\title{
Chiral dynamics in (anti)neutrino-induced pion production off the nucleon
}

\section{De-Liang Yao*}

Institute of Modern Physics, Chinese Academy of Sciences, Lanzhou 730000, China

E-mail: dlyaodimpcas.ac.cn

\section{Luis Alvarez-Ruso}

Departamento de Física Teórica and Instituto de Física Corpuscular (IFIC), Centro Mixto CSIC-UV, Institutos de Investigación de Paterna, E-46071, Valencia, Spain

E-mail: Luis.Alvarez@ific.uv.es

\section{Astrid N. Hiller Blin}

Institut für Kernphysik \& PRISMA Cluster of Excellence, Johannes Gutenberg Universität, D-55099 Mainz, Germany E-mail: Hillerbleuni-mainz.de

\section{J. Vicente Vacas}

Departamento de Física Teórica and Instituto de Física Corpuscular (IFIC), Centro Mixto CSIC-UV, Institutos de Investigación de Paterna, E-46071, Valencia, Spain

E-mail: Manuel.J.Vicentedific.uv.es

\begin{abstract}
The weak pion production off the nucleon is systematically investigated at low energies in a manifestly relativistic chiral perturbation theory in the presence of $\Delta(1232)$ resonances. Most of the involved low-energy constants are previously determined in other processes (or quantities) such as pion-nucleon scattering, while the rest ones are set to be of natural size. As a consequence, the total cross sections for single pion production in three different reaction channels, induced either by neutrino or antineutrino, are predicted. For the neutrino-induced case, our predictions are well consistent with the exiting experimental ANL data except for the $v_{\mu} n \rightarrow \mu^{-} n \pi^{+}$channel. That indicates higher order contributions, behaving as unitarity corrections and extra contact counterterms, are still significant for this channel. In addition, results on neutral-current weak pion production are also discussed. Our chiral representation of weak pion production can be applied to study various low-energy theorems, as well as to analyse pion photo- and electro-production.
\end{abstract}

The 9th International workshop on Chiral Dynamics

17-21 September 2018

Durham, NC, USA

* Speaker. 


\section{Introduction}

Neutrino interaction with matter is at the heart of many interesting and relevant physical processes in astrophysics, nuclear physics, hadron physics and also physics beyond the Standard Model (SM). Neutrino- and antineutrino-induced single pion production off the nucleon account for the dominant contribution to the inclusive (anti)neutrino-nucleus cross section in the relevant energy regime of several experiments. However, at low energies, study of the chiral dynamics of weak single pion productions has been rare. The systematic application of chiral perturbation theory (ChPT) to neutrino-induced pion production beyond leading-order (LO) tree level amplitudes are not well investigated. To our knowledge, it is limited to several low-energy theorems that have been derived for weak pion production, including one-loop corrections, using the heavy-baryon formalism [1]. We report here the first systematic study of weak pion production up to next-tonext-to-leading order (NNLO) in covariant ChPT with nucleons and $\Delta(1232)$. The information gathered in the study in pion production with electromagnetic probes and pion-nucleon scattering within the same framework provides valuable input for weak pion production. By construction, the amplitudes obtained in ChPT fulfill perturbative unitarity and Watson's theorem. As emphasized in Ref. [1], ChPT brings about corrections to the axial current that cannot be derived using PCAC. Furthermore, unlike most phenomenological models, it does not require ad hoc assumptions about the form factors to enforce the (partial) conservation of the (axial) vector current [2]. The predictive power of ChPT calculations is limited to the threshold region but nonetheless they can be very valuable for the neutrino cross-section program [3] as a benchmark for phenomenological models that aim to describe weak pion production in wider energy regions.

In this proceeding, I will review the study of weak pion production in Baryon ChPT (BChPT) based on our works $[4,5]$.

\section{Electroweak interactions in the Standard Model}

After spontaneous breaking of the $S U(2) \times U(1)$ gauge symmetry through a Higgs mechanism, the Lagrangian of electromagnetic and weak interaction in the Standard Model (SM) reads

$$
\mathscr{L}_{\mathrm{EW}}=-e J_{\mathrm{EM}}^{\mu} A_{\mu}-\frac{g}{2 \cos \theta_{W}} J_{\mathrm{NC}}^{\mu} Z_{\mu}-\frac{g}{2 \sqrt{2}} J_{\mathrm{CC}}^{\mu} W_{\mu}^{+}+\text {h.c. },
$$

where $J_{\mathrm{EM}}^{\mu}, J_{\mathrm{NC}}^{\mu}$ and $J_{\mathrm{CC}}^{\mu}$ are the electromagnetic (EM) current, weak neutral current (NC) and charged current (CC), in order. The weak coupling $g$ and the strength of EM interaction $e$ are related by the weak angle $\theta_{W}$ via $\sin \theta_{W}=e / g$. Furthermore, $\cos \theta_{W}=M_{W} / M_{Z}$, where $M_{W}$ and $M_{Z}$ are the masses of the $W^{ \pm}$and $Z$ bosons, respectively.

The interactions between leptons and the gauge bosons are obtained by expressing the corresponding currents in the following form,

$$
\begin{aligned}
J_{\mathrm{EM}}^{\mu} & =\bar{l}_{i} \gamma^{\mu} l_{i}, \quad i=e, \mu, \tau, \\
J_{\mathrm{CC}}^{\mu} & =\bar{v}_{i} \gamma^{\mu}\left(1-\gamma_{5}\right) l_{i}, \\
J_{\mathrm{NC}}^{\mu} & =\frac{1}{2} \bar{l}_{i} \gamma^{\mu}\left(g_{V}-g_{A} \gamma_{5}\right) l_{i}+\frac{1}{2} \bar{v}_{i} \gamma^{\mu}\left(1-\gamma_{5}\right) v_{i},
\end{aligned}
$$


where the NC couplings of charged leptons are given by

$$
g_{V}=-1+4 \sin ^{2} \theta_{W}, \quad g_{A}=-1 .
$$

Likewise, quarks are coupled to the gauge boson through the way

$$
\begin{aligned}
& J_{\mathrm{EM}}^{\mu}=\frac{2}{3} \bar{q}_{u} \gamma^{\mu} q_{u}-\frac{1}{3} \bar{q}_{d} \gamma^{\mu} q_{d}, \\
& J_{\mathrm{NC}}^{\mu}=\bar{q}_{u} \gamma^{\mu}\left[\frac{1}{2}-\left(\frac{2}{3}\right) 2 \sin ^{2} \theta_{W}-\frac{1}{2} \gamma_{5}\right] q_{u}+\bar{q}_{d} \gamma^{\mu}\left[-\frac{1}{2}-\left(-\frac{1}{3}\right) 2 \sin ^{2} \theta_{W}+\frac{1}{2} \gamma_{5}\right] q_{d}, \\
& J_{\mathrm{CC}}^{\mu}=\bar{q}_{u} \gamma^{\mu}\left(1-\gamma_{5}\right) \cos \theta_{C} q_{d},
\end{aligned}
$$

where we have restricted ourselves to the $u$ and $d$ quarks and $\theta_{C} \simeq 13^{\circ}$ is the Cabibbo angle. The above currents in the quark sector can be rewritten in terms of vector and axial-vector currents, which are defined by

$$
\begin{aligned}
& V_{a}^{\mu} \equiv \bar{q} \gamma^{\mu} \frac{\tau_{a}}{2} q, \quad a=0,1,2,3, \\
& A_{a}^{\mu} \equiv \bar{q} \gamma^{\mu} \frac{\tau_{a}}{2} q, \quad a=1,2,3 .
\end{aligned}
$$

Here $\tau_{1-3}$ are Pauli matrices, $\tau_{0}$ is a $2 \times 2$ unit matrix and $q=\left(q_{u}, q_{d}\right)^{T}$. Straightforwardly, one has

$$
\begin{aligned}
J_{\mathrm{EM}}^{\mu} & =\frac{1}{3} V_{0}^{\mu}+V_{3}^{\mu}, \\
J_{\mathrm{NC}}^{\mu} & =\left(1-2 \sin ^{2} \theta_{W}\right) V_{3}^{\mu}-A_{3}^{\mu}-\frac{2}{3} \sin ^{2} \theta_{W} V_{0}^{\mu}, \\
J_{\mathrm{CC}}^{\mu} & =\left[\left(V_{1}-A_{1}\right)+i\left(V_{2}-A_{2}\right)\right] \cos \theta_{C}=\left(V_{+}-A_{+}\right) \cos \theta_{C} .
\end{aligned}
$$

Notice that the weak charged current is coupled to the physical $W^{+}$, however, in practice it is sometimes more convenient to use the Cartesian basis to describe the interaction. Given that $W_{\mu}^{ \pm}=$ $\left(W_{\mu}^{1} \mp i W_{\mu}^{2}\right) / \sqrt{2}$, the interaction in Cartesian basis can be achieved in the way as follows:

$$
\begin{aligned}
J_{\mathrm{CC}}^{\mu} W_{\mu}^{+}+h . c . & =\left(V_{+}^{\mu}-A_{+}^{\mu}\right) \cos \theta_{C} W_{\mu}^{+}+h . c . \\
& =\bar{q}\left[\gamma^{\mu}\left(1-\gamma_{5}\right) \tau_{+} \cos \theta_{C} W_{\mu}^{+}\right] q+h . c . \\
& =\bar{q}\left[\gamma^{\mu}\left(1-\gamma_{5}\right)\left(\tau_{+} W_{\mu}^{+}+\tau_{-} W_{\mu}^{-}\right) \cos \theta_{C}\right] q \\
& =\bar{q}\left[\gamma^{\mu}\left(1-\gamma_{5}\right) \frac{1}{\sqrt{2}}\left(\tau_{1} W_{\mu}^{1}+\tau_{2} W_{\mu}^{2}\right) \cos \theta_{C}\right] q \\
& =\sqrt{2} \cos \theta_{C}\left[\left(V_{1}-A_{1}\right) W_{\mu}^{1}+\left(V_{2}-A_{2}\right) W_{\mu}^{2}\right] \\
& \equiv \sum_{a=1}^{2} J_{\mathrm{CC}}^{\mu, a} W_{\mu}^{a},
\end{aligned}
$$

where the weak charged current with Cartesian index is

$$
J_{\mathrm{CC}, \mathrm{a}}^{\mu}=\sqrt{2} \cos \theta_{C}\left(V_{a}^{\mu}-A_{a}^{\mu}\right)
$$




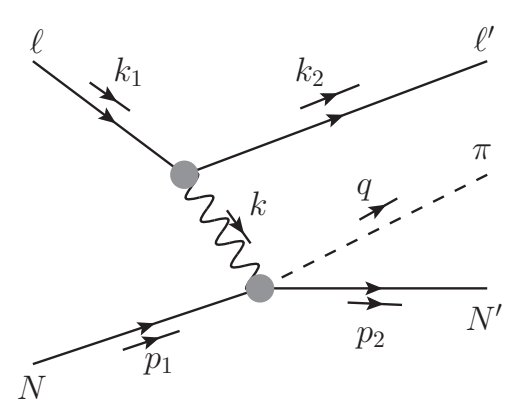

(a)

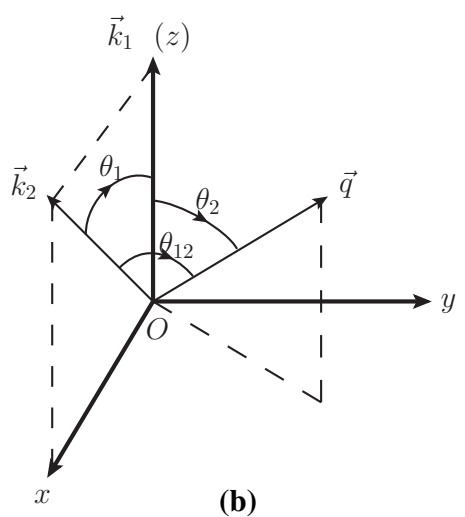

(b)

Figure 1: (a) Single $\pi$ production under one-boson exchange approximation. (b) Reference frame.

\section{Generic description of single pion production off the nucleon}

Under one-boson exchange approximation [see Fig. 1 (a)], the transition matrix element for electroweak single pion production can be written as

$$
\begin{aligned}
& \left\langle\ell^{\prime}\left(k_{2}\right) N^{\prime}\left(p_{2}\right) \pi(q) \mid \ell\left(k_{1}\right) N\left(p_{1}\right)\right\rangle=i(2 \pi)^{4} \delta^{(4)}\left(k_{1}+p_{1}-k_{2}-p_{2}-q\right) \\
& \times N_{X} \frac{-i g^{\mu \nu}}{k^{2}-M_{X}^{2}} N_{X} \underbrace{\left\langle\ell_{L_{V}^{X}}^{\langle}\left|J_{\mathrm{X}}^{\nu}(0)\right| \ell\right\rangle}_{\equiv \mathscr{M}_{X}} \underbrace{\left\langle\pi N^{\prime}\left|J_{\mathrm{X}}^{\mu}(0)\right| N\right\rangle}_{H_{\mu}^{X}} .
\end{aligned}
$$

In the invariant amplitude $\mathscr{M}_{X}$, one has the factor $N_{X}=\left\{-e,-g /\left(2 \cos \theta_{W}\right),-g / 2 \sqrt{2}\right\}$ and the mass $M_{X}=\left\{0, M_{Z}, M_{W}\right\}$ for $X=\{\mathrm{EM}, \mathrm{NC}, \mathrm{CC}\}$, in order. Note that the symbol $\ell$ denote either charge leptons $(\{e, \mu, \tau\} \equiv l$ and their antiparticles $\bar{l})$ or neutrinos $\left(\left\{v_{e}, v_{\mu}, v_{\tau}\right\} \equiv v_{l}\right.$ and their antiparticles $\bar{v}_{l}$ ), so does $\ell^{\prime}$. In the limit of $q^{2} \ll M_{Z, W}^{2}$, the $k^{2}$ term can be neglected. The leptonic and hadronic parts are denoted by $L_{v}^{X}$ and $H_{\mu}^{X}$, respectively. The leptonic amplitude $L_{v}^{X}$ is readily obtained by inserting the leptonic current given in Eq. (2.2). However, it is hard to calculate the hadronic amplitude in QCD perturbatively due to the strength of the strong coupling is extremely large at low energies. Furthermore, quarks are confined to form color-singlet hadrons. Nevertheless, it is doable to perform perturbative calculation of the hadronic amplitude by involving BChPT, which will be discussed in the next section. Before conducting BChPT calculation, we intend to present the structure of the hadronic amplitude, which will simply the calculation greatly.

\subsection{Isospin structure}

Although there are various physical processes, their corresponding amplitudes are related to each other by isospin symmetry. The isospin decomposition of hadronic amplitudes can be universally written as

$$
\left\langle\pi^{b} N^{\prime}\left|J_{\mu}^{a}\right| N\right\rangle=\chi_{f}^{\dagger}\left[\delta^{b a} H_{\mu}^{+}\left(s_{2}, t, t_{1}\right)+i \varepsilon^{b a c} \tau^{c} H_{\mu}^{-}\left(s_{2}, t, t_{1}\right)+\tau^{b} H_{\mu}^{0}\left(s_{2}, t, t_{1}\right)\right] \chi_{i}
$$


where $\chi_{i}$ and $\chi_{f}$ are isospinors of the initial and final nucleon states, respectively. The Mandelstam variables are defined by

$$
\begin{aligned}
& s \equiv\left(k_{1}+p_{1}\right)^{2}, \quad s_{1} \equiv\left(k_{2}+p_{2}\right)^{2}, \quad s_{2} \equiv\left(q+p_{2}\right)^{2}, \\
& t_{1} \equiv\left(k_{1}-k_{2}\right)^{2}, \quad t_{2} \equiv\left(k_{1}-q\right)^{2}, \quad t \equiv\left(p_{1}-p_{2}\right)^{2} \text {. }
\end{aligned}
$$

In view of Eqs. $(2.6,2.7,2.10)$, the isospin even/odd amplitude $H_{\mu}^{ \pm}$and isoscalar amplitude $H_{\mu}^{0}$ can be further expressed in terms of vector and axial-vector amplitudes, i.e., $V_{\mu}^{ \pm}, V_{\mu}^{0}$ and $A_{\mu}^{ \pm}$.

For EM,

$$
\begin{aligned}
H_{\mu}^{ \pm}\left(s_{2}, t, t_{1}\right) & =V_{\mu}^{ \pm}\left(s_{2}, t, t_{1}\right) \\
H_{\mu}^{0}\left(s_{2}, t, t_{1}\right) & =V_{\mu}^{0}\left(s_{2}, t, t_{1}\right)
\end{aligned}
$$

For NC,

$$
\begin{aligned}
H_{\mu}^{ \pm}\left(s_{2}, t, t_{1}\right) & =\left(1-2 \sin ^{2} \theta_{W}\right) V_{\mu}^{ \pm}\left(s_{2}, t, t_{1}\right)-A_{\mu}^{ \pm}\left(s_{2}, t, t_{1}\right) \\
H_{\mu}^{0}\left(s_{2}, t, t_{1}\right) & =\left(-2 \sin ^{2} \theta_{W}\right) V_{\mu}^{0}\left(s_{2}, t, t_{1}\right)
\end{aligned}
$$

For CC,

$$
\begin{aligned}
H_{\mu}^{ \pm}\left(s_{2}, t, t_{1}\right) & =\sqrt{2} \cos \theta_{C}\left(V_{\mu}^{ \pm}\left(s_{2}, t, t_{1}\right)-A_{\mu}^{ \pm}\left(s_{2}, t, t_{1}\right)\right) \\
H_{\mu}^{0}\left(s_{2}, t, t_{1}\right) & =0 .
\end{aligned}
$$

It can be seen from the above equations that once $V_{\mu}^{ \pm, 0}$ and $A_{\mu}^{ \pm}$are known, one can construct all the isospin amplitudes for EM, NC and CC case. Once the functions $H_{\mu}^{ \pm, 0}$ are determined, the hadronic transition amplitudes for the various physical processes can be readily obtained through

$$
H_{\mu}(\text { physical process })=a_{+} H_{\mu}^{+}+a_{-} H_{\mu}^{-}+a_{0} H_{\mu}^{0} .
$$

The physical processes and their corresponding coefficients are collected in Table 1.

\subsection{Lorentz structure}

To proceed, the Lorentz decompositions of $V_{\mu}^{ \pm, 0}$ and $A_{\mu}^{ \pm}$read

$$
\begin{aligned}
V_{\mu}^{ \pm, 0}\left(s_{2}, t, t_{1}\right) & =\sum_{i=1}^{8} \bar{u}_{N^{\prime}}\left(p_{2}\right)\left\{V_{i}^{ \pm, 0}\left(s_{2}, t, t_{1}\right) \mathscr{O}_{\mu, i}^{V}\right\} u_{N}\left(p_{1}\right), \\
A_{\mu}^{ \pm}\left(s_{2}, t, t_{1}\right) & =\sum_{i=1}^{8} \bar{u}_{N^{\prime}}\left(p_{2}\right)\left\{A_{i}^{ \pm}\left(s_{2}, t, t_{1}\right) \mathscr{O}_{\mu, i}^{A}\right\} u_{N}\left(p_{1}\right),
\end{aligned}
$$

with the Lorentz axial-vector operators

$$
\begin{array}{llrl}
\mathscr{O}_{\mu, 1}^{A} & =q_{\mu}, & \mathscr{O}_{\mu, 2}^{A}=p_{1, \mu}, & \mathscr{O}_{\mu, 3}^{A}=p_{2, \mu}, \\
\mathscr{O}_{\mu, 4}^{A}=q q_{\mu}, & \mathscr{O}_{\mu, 5}^{A}=q p_{1, \mu}, & \mathscr{O}_{\mu, 6}^{A}=q p_{2, \mu}, \\
\mathscr{O}_{\mu, 7}^{A}=\gamma_{\mu} q, & \mathscr{O}_{\mu, 8}^{A}=\gamma_{\mu}, &
\end{array}
$$


Table 1: Physical hadronic processes and coefficients.

\begin{tabular}{|c|c|c|}
\hline & Physical Process & $\begin{array}{lll}a_{+} & a_{-} & a_{0} \\
\end{array}$ \\
\hline \multirow{4}{*}{ EM } & $\gamma^{(*)} p \rightarrow p \pi^{0}$ & $\begin{array}{ll}0 & 1\end{array}$ \\
\hline & $\gamma^{(*)} n \rightarrow n \pi^{0}$ & $0-1$ \\
\hline & $\gamma^{(*)} n \rightarrow p \pi^{-}$ & $0-\sqrt{2} \sqrt{2}$ \\
\hline & $\gamma^{(*)} p \rightarrow n \pi^{+}$ & $\begin{array}{ll}0 & \sqrt{2} \\
\sqrt{2}\end{array}$ \\
\hline \multirow{4}{*}{$\mathrm{NC}$} & $Z^{0} p \rightarrow p \pi^{0}$ & \\
\hline & $Z^{0} n \rightarrow n \pi^{0}$ & $0-1$ \\
\hline & $Z^{0} n \rightarrow p \pi^{-}$ & $0-\sqrt{2} \sqrt{2}$ \\
\hline & $Z^{0} p \rightarrow n \pi^{+}$ & $\begin{array}{ll}0 & \sqrt{2} \\
\end{array}$ \\
\hline & $p \rightarrow p \pi^{+} / W^{-} n \rightarrow n \pi^{-}$ & $\begin{array}{lll}1 & -1 & 0\end{array}$ \\
\hline & $n \rightarrow n \pi^{+} / W^{-} p \rightarrow p \pi^{-}$ & 1 \\
\hline & ${ }^{+} n \rightarrow p \pi^{0} / W^{-} p \rightarrow n \pi^{0}$ & $\begin{array}{lll}0 & \sqrt{2} & 0\end{array}$ \\
\hline
\end{tabular}

and Lorentz vector operators

$$
\mathscr{O}_{\mu, i}^{V}=\mathscr{O}_{\mu, i}^{A} \gamma_{5}, \quad i=1, \cdots, 8
$$

The set of vector operators is complete but they are not independent if the conservation of the vector current is imposed. To be specific, there exist two constraints on $V_{i}$ :

$$
\begin{aligned}
k \cdot q V_{1}+k \cdot p_{1} V_{2}+k \cdot p_{2} V_{3}+\left(M_{\pi}^{2}-2 p_{1} \cdot q\right) V_{7}+2 m_{N} V_{8} & =0, \\
k \cdot q V_{4}+k \cdot p_{1} V_{5}+k \cdot p_{2} V_{6}+V_{8} & =0,
\end{aligned}
$$

with $k \equiv k_{1}-k_{2}, M_{\pi}$ and $m_{N}$ being the physical masses of the pion and nucleon, respectively. Knowing the Lorentz structure of the hadronic amplitudes, the following thing is just to determine the unknown scalar functions $V_{i}^{ \pm, 0}\left(s_{2}, t, t_{1}\right)$ and $A_{i}^{ \pm}\left(s_{2}, t, t_{1}\right)$.

\section{Weak pion production in BChPT}

The hadronic part of $\mathrm{CC}$ weak pion production has been calculated within the framework of BChPT up to NNLO in Ref. [4], and later the same formalism has been applied to NC weak pion production in Ref. [5]. Given the working accuracy in Refs. [4, 5], the following chiral Lagrangians are used,

$$
\mathscr{L}_{\text {eff }}=\sum_{i=1}^{2} \mathscr{L}_{\pi \pi}^{(2 i)}+\sum_{j=1}^{3} \mathscr{L}_{\pi N}^{(j)}+\sum_{k=1}^{2}\left[\mathscr{L}_{\pi \Delta}^{(k)}+\mathscr{L}_{\pi N \Delta}^{(k)}\right]
$$

where superscripts represent chiral orders while subscripts denote the relevant degrees of freedom. For clarity, the effective Lagrangian is classified in three parts: the purely pionic sector, the pionnucleon sector and the one involving $\Delta$ resonances. I refer the readers to Refs. [6, 7, 8, 9, 10, 11], for the explicit expression of the Lagrangian. Topologies of tree-level diagrams are shown in 


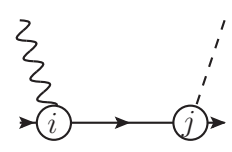

(a)

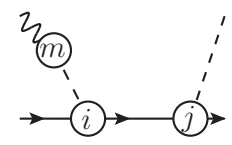

(e)

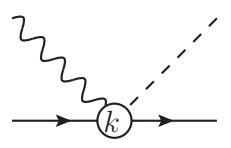

(b)

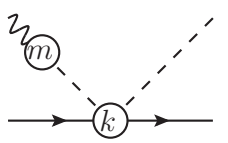

(f)

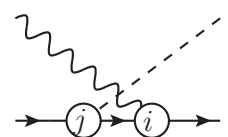

(c)

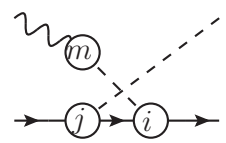

(g)

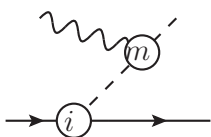

(d)

Figure 2: Topologies of tree-level diagrams. The solid, dashed and wiggled lines represent nucleons, pions and left-hand currents. The letters in the circles mark the possible chiral orders of the vertices. Diagrams with $\Delta$-exchange are obtained by replacing internal nucleon lines by $\Delta$ propagators. Diagrams with mass insertions in the internal pion, nucleon and $\Delta$ propagators are not shown explicitly.

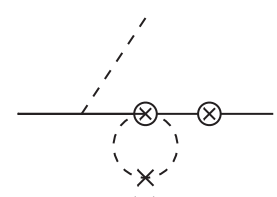

(a)

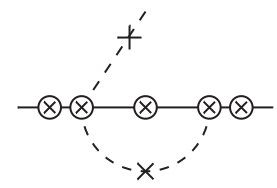

(e)

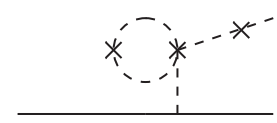

(i)

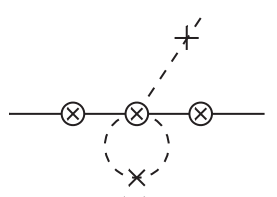

(b)

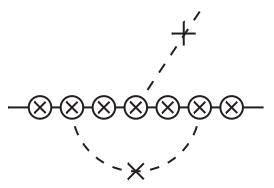

(f)

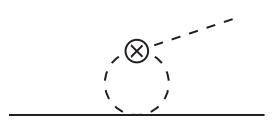

(j)

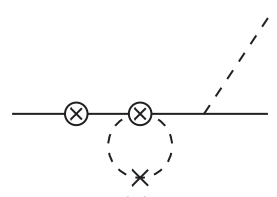

(c)

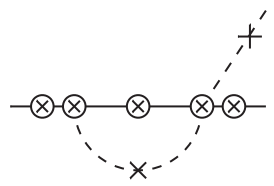

(g)

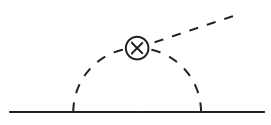

(k)

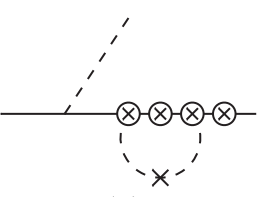

(d)

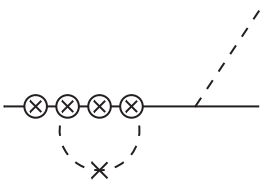

(h)

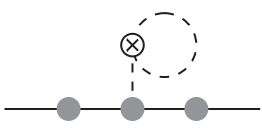

(l)

Figure 3: Topologies from which one-loop diagrams are generated. Topologies leading to corrections on the external pion and nucleon legs are not shown because the corresponding contributions are taken into account by wave-function renormalization. The solid lines represent nucleons, while the dashed ones stand for the pions. Vertices with crosses, circles and grey dots denote positions at which incoming left-hand currents, incoming pions and outgoing pions, respectively, can be inserted. Incoming pions are always coupled to left-hand currents.

Fig. 2, while the ones from which one-loop diagrams can be generated are displayed in Fig. 3. To restore correct power counting, the one-loop hadronic amplitude has been renormalized using the extended-on-mass-shell (EOMS) scheme $[12,13,14]^{1}$. Note that the explicit $\Delta$-resonances are implemented by means of the so-called $\delta$-counting rule proposed in Ref. [18]. Details on the calculations can be found in Ref. [4] and Ref. [5] for CC and NC cases, respectively.

\footnotetext{
${ }^{1}$ See Ref. [15] for its version extended beyond low energies and see also Refs. [16, 17] for further discussion on this topic.
} 


\section{Numerical results of total cross sections}
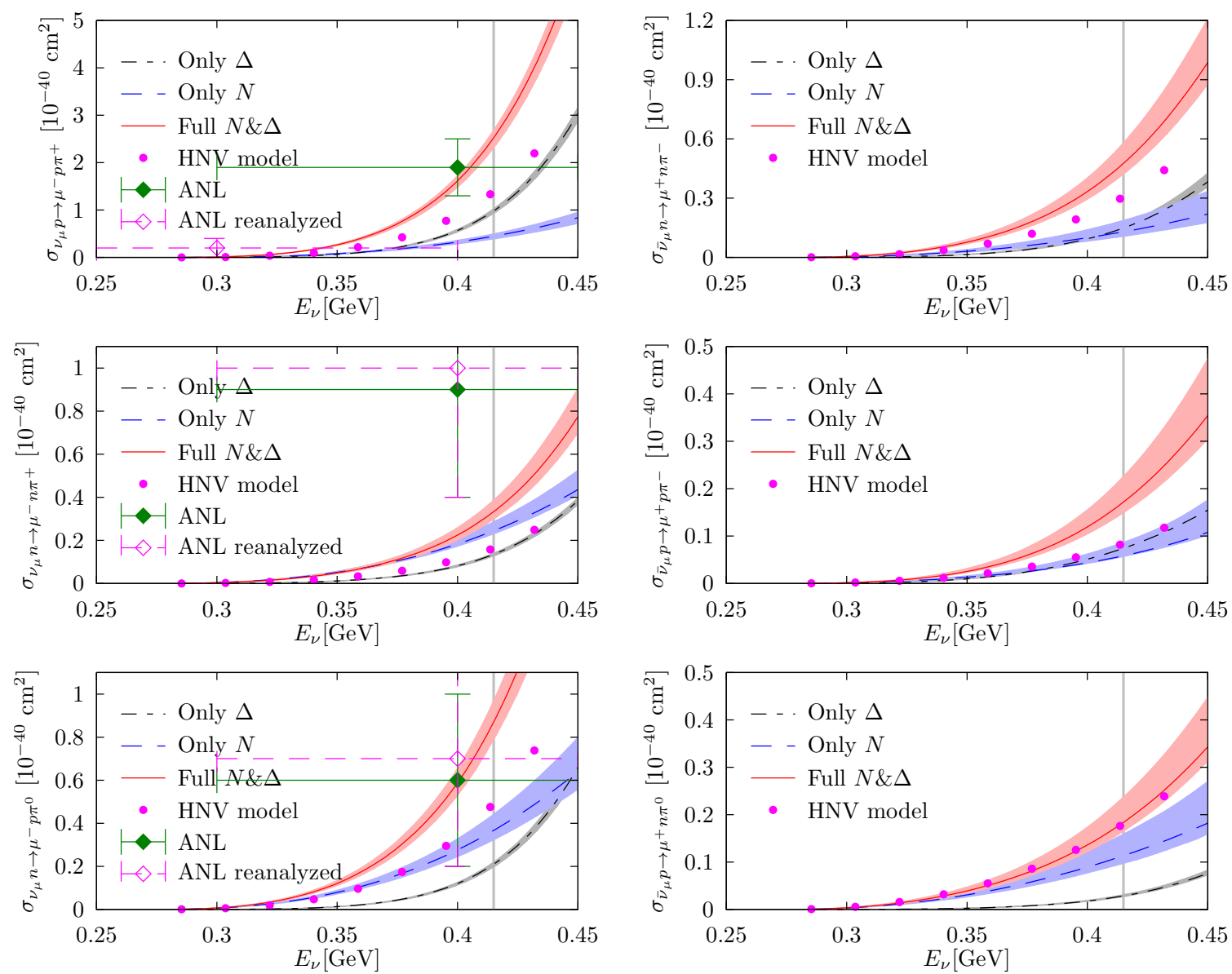

Figure 4: Cross sections for $\mathrm{CC}$ weak pion production. The grey vertical line corresponds to $E_{v}=$ $0.415 \mathrm{GeV}$. Dots correspond to the HNV model [19]. The original ANL data are taken from Refs [20, 21], while the recently reanalyzed ones are from Refs $[22,23]$.

In the center-of-mass (CM) frame of the initial lepton-nucleon (i.e. $\ell-N$ ) pair, the total cross section takes the form

$$
\sigma(s)=\frac{1}{2(4 \pi)^{4} \sqrt{s}\left|\mathbf{k}_{1}\right|} \int_{\omega_{\ell^{\prime}}^{-}}^{\omega_{\ell^{\prime}}^{+}} \mathrm{d} \omega_{v} \int_{\omega_{\pi}^{-}}^{\omega_{\pi}^{+}} \mathrm{d} \omega_{\pi} \int_{-1}^{+1} \mathrm{~d} x_{1} \int_{0}^{2 \pi} \mathrm{d} \phi_{12}|\mathscr{T}|^{2}
$$

where $s \equiv\left(k_{1}+p_{1}\right)^{2}, x_{1} \equiv \cos \theta_{1}$, with $\theta_{1}$ the angle between $\vec{k}_{1}$ and $\vec{k}_{2} ; \phi_{12}$ is the angle between the $\vec{k}_{1} \wedge \vec{k}_{2}$ plane and the $\vec{k}_{2} \wedge \vec{q}$ plane [see Fig. 1 (b)]. Furthermore, $\omega_{\ell^{\prime}}$ and $\omega_{\pi}$ are the energies of 

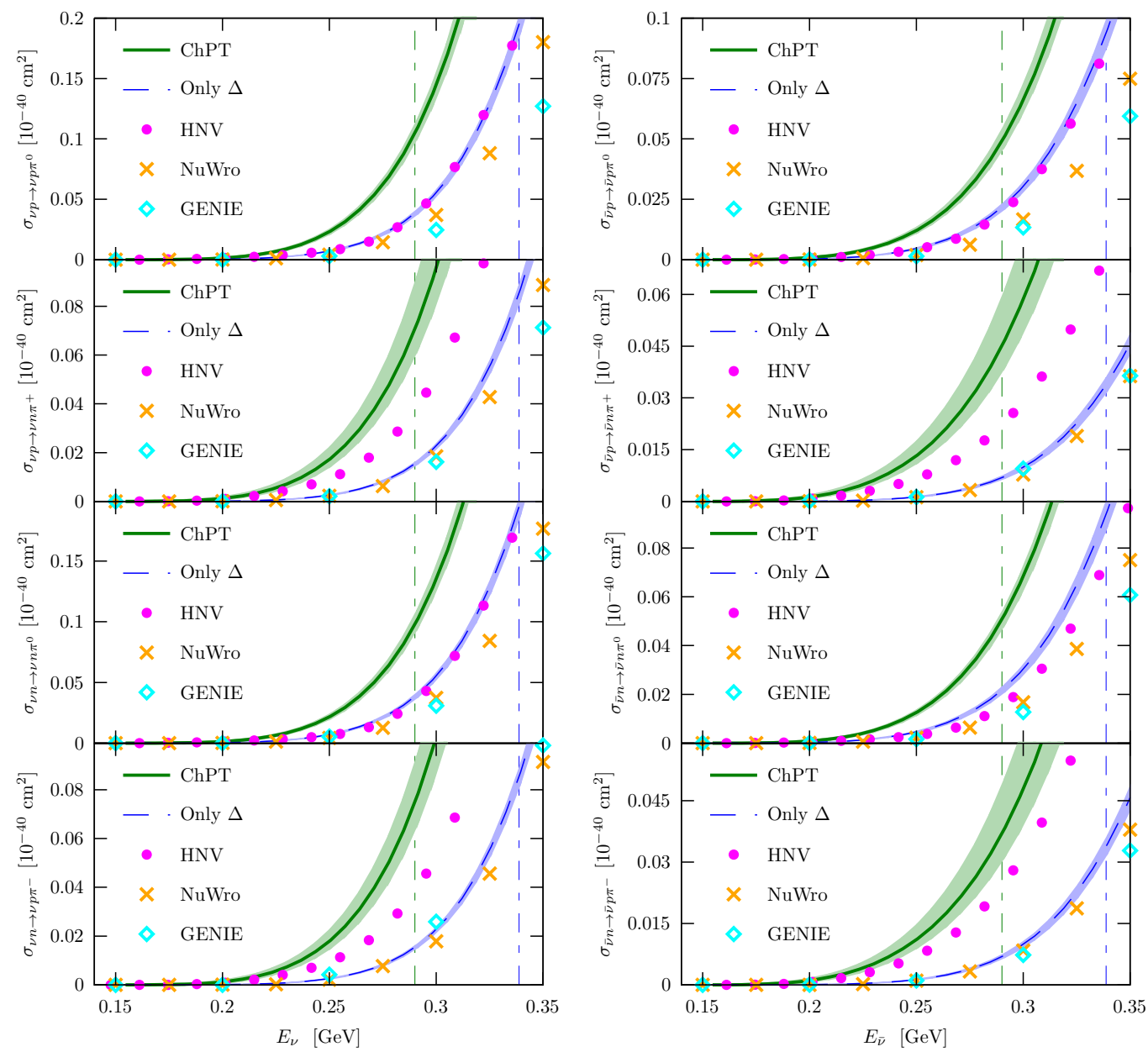

Figure 5: Cross sections for NC weak pion production off the nucleon as a function of the Laboratory neutrino energy. The solid line represents the full $\mathscr{O}\left(p^{3}\right)$ ChPT prediction, while the dashed line stands for the ChPT result obtained with $\Delta$-exchange contributions alone. Our ChPT calculation is expected to be reliable up to the energy marked by the vertical green line. The vertical blue line at a higher energy indicates the energy at which the $\Delta$ pole starts to be reached. The bands account for $1 \sigma$ uncertainties propagated from the errors of the LECs. For comparison, results produced by the HNV model [19], NuWro [24] and GENIE [25] Monte Carlo generators are also shown by dots, crosses and hollow diamonds, respectively.

the outgoing lepton and pion, respectively. Their kinematic limits are given by

$$
\begin{aligned}
& \omega_{\ell^{\prime}}^{-}=m_{\ell^{\prime}}, \quad \omega_{\ell^{\prime}}^{+}=\frac{\left(\sqrt{s}-M_{\pi}\right)^{2}+m_{\ell^{\prime}}^{2}-m_{N}^{2}}{2\left(\sqrt{s}-M_{\pi}\right)}, \\
& \omega_{\pi}^{ \pm}=\frac{1}{2\left(s-2 \omega_{\ell^{\prime}} \sqrt{s}+m_{l}^{2}\right)}\left\{\left(\sqrt{s}-\omega_{\ell^{\prime}}\right)\left(s-2 \omega_{\ell^{\prime}} \sqrt{s}+m_{\ell^{\prime}}^{2}+M_{\pi}^{2}-m_{N}^{2}\right)\right. \\
& \left. \pm\left(\omega_{\ell^{\prime}}^{2}-m_{\ell^{\prime}}^{2}\right) \sqrt{\left[s-2 \omega_{\ell^{\prime}} \sqrt{s}+m_{\ell^{\prime}}^{2}-M_{\pi}^{2}-m_{N}^{2}\right]^{2}-4 M_{\pi}^{2} m_{N}^{2}}\right\},
\end{aligned}
$$

with $\ell^{\prime}$ denoting either the charged or neutral leptons. The unpolarized Lorentz-invariant $\mathscr{M}$-matrix 
squared can be written as

$$
|\mathscr{M}|^{2}=\frac{G_{F}^{2}}{2} L^{\alpha \beta} H_{\alpha \beta},
$$

with the leptonic tensor $\left(\varepsilon_{0123}=-1\right)$,

$$
L_{\alpha \beta}=8\left[k_{1, \alpha} k_{2, \beta}+k_{1, \beta} k_{2, \alpha}-g_{\alpha \beta} k_{1} \cdot k_{2} \pm i \varepsilon_{\alpha \beta \rho \sigma} k_{1}^{\rho} k_{2}^{\sigma}\right] .
$$

The plus (minus) sign corresponds to the neutrino- (antineutrino-) induced reactions, respectively. In terms of the hadronic amplitudes $H_{\alpha}$ introduced in Sec. 3, the hadronic tensor reads

$$
H_{\alpha \beta}=\frac{1}{2} \operatorname{Tr}\left[\left(\not p_{1}+m_{N}\right) \tilde{H}_{\alpha}\left(\not p_{2}+m_{N}\right) H_{\beta}\right],
$$

where $\tilde{H}_{\alpha}=\gamma_{0} H_{\alpha}^{\dagger} \gamma_{0}$.

In numerical computation, most of the required low-energy constants (LECs) are known, as they have been obtained in the analysis of other processes or physical quantities. We take their values from the studies of $\pi N$ scattering [26, 27, 11] and the axial radius of the nucleon [28], which used the EOMS scheme as in the present calculation. The remaining LECs are assumed to be of natural size. Cross sections from (anti)neutrino-induced CC and NC pion production are shown in Fig. 4 and Fig. 5, respectively.

\section{Summary}

I have reviewed the studies of $\mathrm{CC}$ and $\mathrm{NC}$ weak pion production off the nucleon in covariant ChPT with explicit $\Delta(1232)$ up to $\mathscr{O}\left(p^{3}\right)$ following the $\delta$-counting rule. The amplitudes have been renormalized using the EOMS scheme.

On the one hand, it has been found that the predictions on CC cross sections are consistent with the few existing experimental ANL data for the neutrino-induced processes except $v_{\mu} n \rightarrow \mu^{-} n \pi^{+}$. This might indicate that higher-order contributions are still relevant for this channel as suggested by the more phenomenological study of Ref. [29]. Lacking a full calculation, such higher-order contributions might be approximated by unitarity corrections or some extra contact counterterms.

On the other hand, for the $\mathrm{NC}$ case, in the incoming-neutrino energy interval in which the calculation is expected to be valid, predictions for the $\Delta$ excitation mechanism conform well with the corresponding output from the widely used NuWro and GENIE neutrino event generators. However, for all the channels the full ChPT results are significantly larger due to the systematic and model-independent inclusion of non-resonant terms. This observation implies that a precise description of low-energy weak pion production requires a realistic account of non-resonant amplitudes.

\section{References}

[1] V. Bernard, N. Kaiser and U. G. Meißner, Phys. Lett. B 331, 137 (1994) [hep-ph/9312307].

[2] J. H. Koch, V. Pascalutsa and S. Scherer, Phys. Rev. C 65, 045202 (2002) [nucl-th/0108044].

[3] L. Alvarez-Ruso et al., Prog. Part. Nucl. Phys. 100, 1 (2018) [arXiv:1706.03621 [hep-ph]]. 
[4] D. L. Yao, L. Alvarez-Ruso, A. N. Hiller Blin and M. J. Vicente Vacas, Phys. Rev. D 98, no. 7 , 076004 (2018) [arXiv:1806.09364 [hep-ph]].

[5] D. L. Yao, L. Alvarez-Ruso and M. J. Vicente Vacas, arXiv:1901.00773 [hep-ph].

[6] J. Gasser and H. Leutwyler, Annals Phys. 158, 142 (1984).

[7] J. Gasser, M. E. Sainio and A. Svarc, Nucl. Phys. B 307, 779 (1988). doi:10.1016/0550-3213(88)90108-3

[8] N. Fettes, U. G. Meißner, M. Mojzis and S. Steininger, Annals Phys. 283, 273 (2000) Erratum: [Annals Phys. 288, 249 (2001)] [hep-ph/0001308].

[9] T. R. Hemmert, B. R. Holstein and J. Kambor, Phys. Lett. B 395, 89 (1997) [hep-ph/9606456].

[10] T. R. Hemmert, B. R. Holstein and J. Kambor, J. Phys. G 24, 1831 (1998) [hep-ph/9712496].

[11] D. L. Yao, D. Siemens, V. Bernard, E. Epelbaum, A. M. Gasparyan, J. Gegelia, H. Krebs and U. G. Meißner, JHEP 1605, 038 (2016) [arXiv:1603.03638 [hep-ph]].

[12] J. Gegelia and G. Japaridze, Phys. Rev. D 60, 114038 (1999) [hep-ph/9908377].

[13] J. Gegelia, G. Japaridze and X. Q. Wang, J. Phys. G 29, 2303 (2003) [hep-ph/9910260].

[14] T. Fuchs, J. Gegelia, G. Japaridze and S. Scherer, Phys. Rev. D 68, 056005 (2003) [hep-ph/0302117].

[15] E. Epelbaum, J. Gegelia, U. G. Meißner and D. L. Yao, Eur. Phys. J. C 75, no. 10, 499 (2015) [arXiv:1510.02388 [hep-ph]].

[16] V. Bernard, Prog. Part. Nucl. Phys. 60, 82 (2008) [arXiv:0706.0312 [hep-ph]].

[17] L. Geng, Front. Phys. (Beijing) 8, 328 (2013) [arXiv:1301.6815 [nucl-th]].

[18] V. Pascalutsa and D. R. Phillips, Phys. Rev. C 67, 055202 (2003) [nucl-th/0212024].

[19] E. Hernandez, J. Nieves and M. Valverde, Phys. Rev. D 76, 033005 (2007) [hep-ph/0701149].

[20] G. M. Radecky et al., Phys. Rev. D 25, 1161 (1982) Erratum: [Phys. Rev. D 26, 3297 (1982)].

[21] S. J. Barish et al., Phys. Rev. D 19, 2521 (1979).

[22] C. Wilkinson, P. Rodrigues, S. Cartwright, L. Thompson and K. McFarland, Phys. Rev. D 90, no. 11, 112017 (2014) [arXiv:1411.4482 [hep-ex]].

[23] P. Rodrigues, C. Wilkinson and K. McFarland, Eur. Phys. J. C 76, no. 8, 474 (2016) [arXiv:1601.01888 [hep-ex]].

[24] C. Juszczak, J. A. Nowak and J. T. Sobczyk, Nucl. Phys. Proc. Suppl. 159, 211 (2006) [hep-ph/0512365].

[25] C. Andreopoulos et al., Nucl. Instrum. Meth. A 614, 87 (2010) [arXiv:0905.2517 [hep-ph]].

[26] J. M. Alarcon, J. Martin Camalich and J. A. Oller, Annals Phys. 336, 413 (2013) [arXiv:1210.4450 [hep-ph]].

[27] Y. H. Chen, D. L. Yao and H. Q. Zheng, Phys. Rev. D 87, 054019 (2013) [arXiv:1212.1893 [hep-ph]].

[28] D. L. Yao, L. Alvarez-Ruso and M. J. Vicente-Vacas, Phys. Rev. D 96, no. 11, 116022 (2017) [arXiv:1708.08776 [hep-ph]].

[29] E. Hernandez and J. Nieves, Phys. Rev. D 95, no. 5, 053007 (2017) [arXiv:1612.02343 [hep-ph]]. 